\title{
试析 BIM 技术在智慧建筑中的应用
}

\author{
金阳
}

河南省第二建设集团有限公司

DOI:10.18686/bd.v2i8.1560

[摘 要] 当前,智慧建筑已经成为建筑行业发展中的一个重要趋势, BIM 技术在建筑工程建设全过程中具有提高工程效益 的作用。当前 BIM 在我国智慧建筑中的应用还处于高速发展的阶段,因此对 BIM 技术在智慧建筑中的应用进行分析和研 究也有着十分深远的意义。

[关键词] BIM 技术; 智慧建筑; 应用

现阶段,城市发展中出现了能源、环境、交通等方面的 诸多问题,阻碍了城市的可持续发展。降低项目成本风险,提 高工程建设的效率成为了人们高度关注的问题。而 BIM 技 术在智慧城市的建设中的应用,可以说是一个全新的尝试。

\section{BIM 技术与智慧建筑的概述}

BIM 技术主要是根据建筑工程的多个参数建立的一种 三维建筑模型,该技术采用数字信息仿真手段,对与建筑物 相关的真实信息进行数据化处理。其在建筑工程设计、建 造、管理中有着较为广泛的应用。在建筑的整个生命周期均 可实现信息共享、效果的可视化,同时管理的精细化水平也 得到了显著的提升。BIM 技术在很多建筑工程中都得到了 有效的应用,一方面加快了工程进度,另一方面也提高了施 工质量, 有效控制了成本投人, 因此其对建筑行业的发展也 有着十分重要的意义。

我国经济发展中十分提倡可持续发展理念, 因此建筑 行业发展中,智慧建筑也成为了新的发展趋势。智慧建筑是 对传统智能建筑的重要发展。传统智能建筑以建筑物作为 重要的基础和前提, 同时在系统应用的过程中也更好地融 合了架构、系统、应用、管理和优化等多个功能,充分展现了 建筑的智慧性。该建筑可让人与建筑和环境三者间形成和 谐的关系, 从而为人们提供一个更为安全、舒适、便利的生 活环境。

智慧建筑建设和发展的过程中, 应用系统集成的方式 将 $5 \mathrm{~A}$ 功能与现代建筑艺术充分地融合在一起, 从而更好地 体现了智慧建筑的优势。智慧建筑建设主张以人为本, 服务 对象也从传统的物业转变为用户, 利用高科技手段实现对 城市运行核心系统关键信息的收集、分析和整理,从而为多 个子系统之间的互联互通创造了良好的条件, 有效减少了 能源消耗, 控制了环境污染。

\section{BIM 技术在智慧建筑中的应用}

2.1 在智慧建筑规划中的应用

当前城市规模日益扩大, 但城市交通环境信息等处于 无序的状态, 这也在一定程度上加大了城市规划预测的复 杂性。对此我们必须做好城市综合信息的处理工作,同时还 要建立一个科学完善的信息数据中心, 进而分析和解决城
市中的问题。

采用 BIM 技术可以在保证地上建筑和地下管线得到 有效优化处理的基础上，对地下管线进行信息化监测和处 理。在对地上项目信息进行分析后,创建一个能够覆盖地上 和地下构筑物的信息模型, 数据模型具有非常强的综合性 特征, 在数据分析的过程中展示出了非常明显的优势, 为城 市规划发展中数据的分析提供了强大的技术支持。

城市信息模型和数据系统可以应用在多个规划管理环 节。此外其还能实现点线面的有机结合。采用城市大数据技 术,使城市的建设、规划、管理质量及效率都得到了显著的 提升, 且该技术对城市发展中的多个模块都能做出智能化 的响应。由于 BIM 技术的应用,大部分项目都能够创建一个 科学完善的 BIM 信息系统, 数据库中的数据是数字化城市 各类应用中的基础数据, 其在城市智慧化运行和管理的过 程中起到了非常重要的作用,如对城市区域规划、地下工程 和过江隧道等工程的多种信息进行有效监测和分析, 而且 其在多个领域的应用, 也推动了城市信息集中化和城市智 慧化的建设与发展。

另外，城市地下管道的规划建设具有十分显著的复杂 性特征, 隐蔽工程一旦出现问题对其他多个环节都会产生 十分显著的影响。BIM 技术的应用和发展能够十分有效地 解决工程规划和建设过程中存在的问题与不足。采用 BIM 技术能够创建更为完善的城市地下管网信息库, 从而有效 保证城市管网的后期运营。而采用 BIM 技术进行地下管网 信息系统处理，则能够更好地提高城市地下空间应用的可 靠性和便捷性。同时系统自身也具备非常好的信息集成以 及数据分析功能, 并以此推动城市建设质量和水平的显著 提升。

2.2 在智慧建筑设计施工中的应用

在智慧建筑设计工作中, 应用 BIM 技术创建虚拟的建 筑模型,可以更加清晰地确定设计方案,且,其转变了传统二 维设计模式,采用三维立体设计使设计效果更为直观。为建 筑功能的选择和科学决策提供了很好的条件。采取三维展 示的方式展现设计方案, 能够提高建筑性能及成本预测的 科学性及合理性。 
此外,应用 BIM 技术可将非图形数据输人到软件当中, 从而有效评估建筑的重要参数, 为设计方案的优化和改良 提供了理论和数据支持。传统设计方式主要依靠人工来完 成设计方案的确定, 因此也容易由于设计师的失误而出现 比较明显的误差和遗漏。应用 BIM 技术后, 可建立虚拟模 型。可以对设计的方案以及相关参数进行及时调整, 在提高 设计质量的同时也大大加快了设计的速度。并且不同专业 之间也能更好地协调与配合, 摆脱了传统设计模式中多个 专业在同一个模型上协同工作的局限, 大大提高了设计的 质量与效率。

智慧建筑施工中,BIM 技术基础上设计出的图纸, 显著 提高了设计的科学性与合理性, 有效控制了设计变更问题, 最后有效保证了施工进度。故此, 在项目施工时, 应积极创建 信息化管理系统, 使参建各方能够在相同的信息系统中完 成数据的收集、整理、汇总和分析等多项工作内容。保证多 个单位协调作业的正常进行。

除此之外,数据信息传递的速度,工程技术以及造价问 题的处理速度和工程建设的进度都得到了有效的提升。工 程造价验算时,可借助 BIM 技术构建的虚拟模型,完成工程 量的统计和验算工作, 该技术可增强工程量计算的可靠性 与准确性。而且采用三维建模方式进行施工,施工图纸变更 几率明显降低,在有效控制了工程成本投入的同时,也大大 强化了工程的建设质量。

2.3 在智慧建筑运营和维护中的应用

在 BIM 基础上建设起来的智能建筑，其可设立科学的 设计模型、施工模型和竣工模型, 进而有效提高工程运行和 设备管理维护信息的准确性和可靠性。与此同时,在项目竣 工并投人使用后, 还能以竣工模型为基础开展项目运营工 作。在项目设计与施工中,只要将 BIM 建筑模型信息传递到 建筑运营平台, 就可以创建三维立体化模型, 既保证了建筑 安装设备的运行状态,也维护了信息的有效查询。

BIM 技术的应用还能够对建筑的综合性能以及能源消 耗等多种情况予以科学分析和评估, 或者也可对建筑运营 阶段设备运行效率加以监测和控制。另外,BIM 运营平台数 据处理的过程中, 也可以对各种可能发生的安全问题和自 然灾害进行可视化模拟, 根据实际情况制定科学的应急处 理方案。若出现事故,则可依照监控数据分析模拟得出的最 佳线路,完成人员的疏散工作,进而有效降低事故造成的损 失。

3 实际应用效果

3.1 工程概况
某项目是商业办公一体化景观办公建筑，建筑自身较 为复杂, 因此设计和施工中需要处理多项工作, 这就加大了 设计和施工的难度。该项目位于 $\mathrm{A}$ 市的核心地带,总占地面 积为 12.3 万平方米, 建筑面积为 63 万平方米,办公区域的 面积为 42 万平方米。在该项目的设计、施工和运行维护等 多个阶段均应用了 BIM 技术。

\section{2 应用效果}

在项目的筹建和及运行维护中, 采用 BIM 技术对工程 项目开展了高水平的信息化管理, 大大提高了项目的施工 效率和施工质量, 同时工程的经济效益也得到了更好地展 现。缩短了工程的建设周期,完善了工程的性能。

采用 $3 \mathrm{D}$ 模拟的方式有效控制了施工现场中工艺方面 的矛盾,提高了工程量计算的自动化水平。在机电安装环节 以及钢结构优化设计的应用管理过程中, 显著缩短了工程 的设计周期,而且工程的设计质量也得到了优化。再者,相关 人员利用 BIM 技术开展了全面的技术讨论和方案比对,一 方面提高了工程设计和建设的质量及效率, 另一方面也更 好地保证了多个专业的协调与合作。在事故发生前及时发 现工程中的不足,降低了工人的劳动强度, 同时也大大地节 约了工程成本,有效保证了建筑工程的质量及安全。不仅如 此,BIM 技术还可实现工程实物量的计算, 与人工计算相比, 其精度更高。同样值得注意的是,在对比合同及实际施工消 耗、分项单价以及分项单价与合价的基础上,也可保证项目 成本风险控制的质量与效果。

\section{4 结语}

建筑行业的发展对推动经济发展有着十分重要的意 义。现场施工的不足拖慢了建筑业生产的速度。当前, 建筑 行业内部的竞争十分激烈, 建筑企业的利润也越来越低,工 程投资、工期以及质量控制管理工作难度越来越大。针对这 一状况,应积极采用 BIM 技术,推动建筑智能化与智慧化的 发展,通过智慧化建筑的应用,可有效减少建筑的能源消耗, 在缩短工期, 保护环境的同时也能显著降低工程的造价, 为 我国建筑行业做出贡献。

\section{参考文献:}

[1]王玉卿,穆华倩.BIM 技术在智慧建筑中的应用 [J]. 智能建筑电气技术, 2016,10(05):53-57.

[2]徐蕾.试析 BIM 在智能建筑设计中的实施要点 [J]. 环球市场,2016,(24):159-159.

[3]张翔. BIM 技术在智慧建筑中的应用 [ J]. 厦门科技, 2017,(05):46-48. 